\title{
Evaluation of the acute and chronic toxicity of the jiangu capsules
}

\author{
ZENGMEI LAN $^{1,2}$, LIPING WANG ${ }^{1}$, ZHAOYANG CHONG $^{1}$, \\ GUANGDE YANG $^{3}$, XIAOJIANG YU ${ }^{1}$, LINA CHEN ${ }^{1}$ and JUN ZHOU ${ }^{1}$
}

\begin{abstract}
${ }^{1}$ Department of Pharmacology, School of Basic Medical Sciences, Xi'an Jiaotong University Health Science Center, Xi'an, Shaanxi 710061; ${ }^{2}$ Department of Software Engineering School of Computer Science and Communication Engineering, Guangxi University of Science and Technology, Liuzhou, Guangxi 545006; ${ }^{3}$ Department of Pharmaceutical Analysis, School of Pharmacy, Xi'an Jiaotong University Health Science Center, Xi'an, Shaanxi 710061, P.R. China
\end{abstract}

Received October 10, 2015; Accepted October 28, 2016

DOI: $10.3892 / \mathrm{etm} .2017 .5341$

\begin{abstract}
Saussureae involucratae Flos is the primary component of the jiangu capsule, which also consists of 12 types of natural medicine. It is a traditional Chinese medicine used to treat a number of conditions including arthralgia, cold and sore muscles, stiff limbs, inconvenient flexion and activity limitation caused by different types of osteoarthritis. The present study was performed to assess the safety of the jiangu capsule following acute and chronic administration in mice and rats. In the acute study, mice were treated with the jiangu capsule orally at $16 \mathrm{~g} / \mathrm{kg} / \mathrm{day}$, the highest dose, for 14 days. In the chronic oral toxicity study, rats were treated with 2,4 and $8 \mathrm{~g} / \mathrm{kg} /$ day of the jiangu capsule for 6 months. The general condition, body weight changes, food and water consumption, hematological and biochemical parameters, urinalysis, systematic anatomy and organ weights of the rats were monitored and histopathological examination was performed at the end of 3 and 6 months' daily administration, and following 14 days of withdrawal. There were no signs of toxicity in the general condition and body weight of the mice was not significantly affected by the treatment. Furthermore, necropsy findings for the animals in the acute and chronic toxicity studies demonstrated that no mortalities had occurred. The results of the current study indicate that treatment with the jiangu capsule did not induce mortality or any detectable abnormalities in mice and rats.
\end{abstract}

\section{Introduction}

Traditional Chinese medicine (TCM) has been used for a long time and has a large market value as a medicinal product.

Correspondence to: Professor Jun Zhou or Professor Lina Chen, Department of Pharmacology, School of Basic Medical Sciences, Xi'an Jiaotong University Health Science Center, 76 Yanta West Road, Xi'an, Shaanxi 710061, P.R. China

E-mail: jzhou829@126.com

E-mail: chenlin@xjtu.edu.cn

Key words: jiangu capsule, hematological parameters, biochemical parameters, organ weights, histopathological examination
Saussureae involucratae Flos, also known as the blood lotus or thumb flower, is a well-known traditional Chinese medicine used to treat a number of different conditions, including rheumatoid arthritis, lower abdominal pain in women, retained placenta and amenorrhea (1-3). Saussureae involucratae Flos grows in gravel near the snow line of mountains at $4800-5800 \mathrm{~m}$ above sea level, in areas that include Sinkiang, Qinghai, Tibet and Gansu (4,5). It is a typical alpine plant and a rare Chinese medicinal herb with resistance to the cold, which is hard to obtain. Saussureae involucratae Flos contains flavonoids, alkaloids and sesquiterpenoids (6), and has been demonstrated to dispel cold and remove dampness, stimulate menstrual flow and promote blood circulation (6). The primary component of jiangu is Saussureae involucratae Flos, however it also consists of 12 types of natural medicine, listed in Table I. These natural components include Punica granatum L. Semen (7), Meconopsis horridula (8), Gentianae Macrophyllae Radix Flos (9), Tinospora sinensis (10), Caryophylli Flos (11), Amomi Fructus Rotundus and Cassiae Semenv (12) that are known to be potent in promoting blood circulation to remove blood stasis and preventing osteoarthritis. In addition, Cinnamomi Cortex (13), Zingiberis Rhizoma, Carthami Flos (14) and Polygonati Rhizoma (15) are components of jiangu with anti-inflammatory and analgesic effects. The jiangu capsule acts to promote blood circulation, eliminate cold and dampness from the body and reduce inflammation. Therefore, it has been proposed as a novel treatment of rheumatoid arthritis and different types of osteoarthritis (16).

Limited information exists on the safety of treatment with jiangu; therefore the present study assessed the toxicity of acute and chronic oral doses of jiangu. The potential toxicity following 14 days or 6 months of repeated oral administration was assessed in mice or rats, respectively, in order to assess the safety of the jiangu capsule.

\section{Materials and methods}

Jiangu capsule preparation. The jiangu capsule was prepared using 12 herbs, which are listed in Table I with the purchasing details. Cinnamomi Cortex, Zingiberis Rhizoma, Caryophylli Flos, Amomi Fructus Rotundus, respectively, were smashed through an 80 mesh sieve and then mixed with extracts I and II. The compounds Saussureae involucratae 
Table I. Composition of the jiangu capsule.

\begin{tabular}{|c|c|c|c|c|}
\hline Chinese name & Latin name & Scientific name & Supplier details & Ratio (\%) \\
\hline Xue lian hua & $\begin{array}{l}\text { Saussureae involucratae } \\
\text { Flos }\end{array}$ & $\begin{array}{l}\text { Saussurea medusa } \\
\text { Maxim }\end{array}$ & $\begin{array}{l}\text { Tibet Autonomous Region Medicine } \\
\text { Company, Lhasa, China }\end{array}$ & 20.41 \\
\hline Qin jiao hua & $\begin{array}{l}\text { Gentianae Macrophyllae } \\
\text { Radix Flos }\end{array}$ & $\begin{array}{l}\text { Gentiana Straminea } \\
\text { Maxim }\end{array}$ & $\begin{array}{l}\text { Tibet Autonomous Region Drug } \\
\text { Mill, Lhasa, China }\end{array}$ & 13.62 \\
\hline Shi liu zhi & $\begin{array}{l}\text { Punica granatum } \\
\text { L. Semen }\end{array}$ & Punica granatum $L$ & $\begin{array}{l}\text { Tibet Autonomous Region Medicine } \\
\text { Company, Lhasa, China }\end{array}$ & 20.41 \\
\hline Rou gui & Cinnamomi Cortex & $\begin{array}{l}\text { Cinnamomum cassia } \\
\text { Presl }\end{array}$ & $\begin{array}{l}\text { Xi'an Chinese medicine yinpian } \\
\text { factory co., LTD, Xi'an, China }\end{array}$ & 4.08 \\
\hline Gan jiang & Zingiberis Rhizoma & Zingiber officinale Rosc & $\begin{array}{l}\text { Xi'an Chinese medicine yinpian } \\
\text { factory co., LTD, Xi'an, China }\end{array}$ & 6.80 \\
\hline Ding xiang & Caryophylli Flos & $\begin{array}{l}\text { Eugenia caryophyllata } \\
\text { Thunb }\end{array}$ & $\begin{array}{l}\text { Xi'an Chinese medicine yinpian } \\
\text { factory co., LTD, Xi'an, China }\end{array}$ & 3.40 \\
\hline Hong hua & Carthami Flos & Carthamus tinctorius $L$ & $\begin{array}{l}\text { Xi'an Chinese medicine yinpian } \\
\text { factory co., LTD, Xi'an, China }\end{array}$ & 3.40 \\
\hline Kuan jin teng & Tinospora sinensis & $\begin{array}{l}\text { Tinospora sinensis (Lour) } \\
\text { Merr. }\end{array}$ & $\begin{array}{l}\text { Tibet Autonomous Region Medicine } \\
\text { Company, Lhasa, China }\end{array}$ & 6.80 \\
\hline Dou kou & $\begin{array}{l}\text { Amomi Fructus } \\
\text { Rotundus. }\end{array}$ & $\begin{array}{l}\text { Amonum kravanh Pierre } \\
\text { ex Gugnep }\end{array}$ & $\begin{array}{l}\text { Xi'an Chinese medicine yinpian } \\
\text { factory co., LTD, Xi'an, China }\end{array}$ & 4.08 \\
\hline Jue ming zi & Cassiae Semen & Cassia obtusifolia $L$ & $\begin{array}{l}\text { Xi'an Chinese medicine yinpian } \\
\text { factory co., LTD, Xi'an, China }\end{array}$ & 6.80 \\
\hline Huang jing & Polygonati Rhizoma & $\begin{array}{l}\text { Polygonatum kingianum } \\
\text { coll.et Hemsl }\end{array}$ & $\begin{array}{l}\text { Xi'an Chinese medicine yinpian } \\
\text { factory co., LTD, Xi'an, China }\end{array}$ & 6.80 \\
\hline $\begin{array}{l}\text { Duo ci lv } \\
\text { rong gao }\end{array}$ & Meconopsis horridula & $\begin{array}{l}\text { Meconopsis horridula } \\
\text { Hook.f.et Thoms }\end{array}$ & $\begin{array}{l}\text { Tibet Sunbara shenshui Drug } \\
\text { Mill, Lhasa, China }\end{array}$ & 3.40 \\
\hline
\end{tabular}

Flos, Gentianae Macrophyllae Radix Flos, Punica granatum L. Semen, Tinospora sinensis, Cassiae Semen, Carthami Flos and Meconopsis horridula underwent reflux extraction in ten parts $65 \%$ ethanol to one part powder for $1 \mathrm{~h}$ at $60^{\circ} \mathrm{C}$. This was repeated twice under low pressure using the filtrate from the previous extraction to produce extract I at a relative density of 1.30-1.33. Polygonati Rhizoma and the dregs from the previous extraction were mixed and decocted twice in $10 \mathrm{x}$ water. To produce extract II, the filtrate was combined without the dregs under low pressure at $60^{\circ} \mathrm{C}$, to produce a relative density of 1.30-1.33. Extract I and II were collected and dried at low pressure at a temperature of $50-60^{\circ} \mathrm{C}$ prior to mixing with Cinnamomi Cortex, Zingiberis Rhizoma, Caryophylli Flos and Amomi Fructus Rotundus. The granule was produced following the use of a 14-mesh sieve with $80 \%$ ethanol, used to soften the material and the 16-mesh sieve was used to prepare the granule. This compound was enclosed into a size 0 capsule $(0.30 \mathrm{~g}$ per capsule). A patent for the herbal formula has been applied for from the State Intellectual Property Office of the P.R. China (Beijing, China) under the ID: 201510149957.7.

Experimental animals and housing conditions. A total of 30 ICR mice (15 females and 15 males, 4 weeks old), weighing 18-22 g, were used for the acute toxicity study. The chronic toxicity test was conducted with a total of 120 4-week old Sprauge-dawley (SD) rats at a gender ratio of 50:50 weighing
80-100 g. All animals were obtained from the Experimental Animal Center of Xi'an Jiaotong University (Xi'an, China). Animals were kept in colony cages; 10 rats or 5 mice per cage, under the following laboratory conditions in a ventilated room: $18-25^{\circ} \mathrm{C}, 35-50 \%$ humidity and a $12 \mathrm{~h}$ light dark cycle. Animals were fed a standard commercial diet and tap water. The experimental protocol was undertaken in accordance with the Guidelines of Care and Use of Laboratory Animals issued by the Chinese Council on Animal Research and the Guidelines of Animal Care (17). The present study was approved by the Animal Ethical Committee of Xi'an Jiaotong University (Xi'an, China).

Acute toxicity study. The highest dose method was adopted for the acute toxicity test (18). Doses were calculated by evaluating the dissolving capacity of the jiangu capsule in distilled water. ICR mice were randomly assigned to one of two groups: A control group of 10 mice and a medicated group of 20 mice ( 5 per cage, segregated by gender). The medicated group received a $40 \%$ concentration of Jiangu capsule, $0.4 \mathrm{ml} / 10 \mathrm{~g} /$ day $(16 \mathrm{~g} / \mathrm{kg} /$ day) by oral gavage (treatment was administered twice in $24 \mathrm{~h}$ with an interval of $6 \mathrm{~h}$ between doses). The control group was administered distilled water $(0.4 \mathrm{ml} / 10 \mathrm{~g} /$ day). Mice had ad libitum access to food and water. The mice were observed for general behavior changes, toxicity and mortality continuously for $4 \mathrm{~h}$ after dosing, intermittently during a $24 \mathrm{~h}$ period and then kept for a further 14 days. 

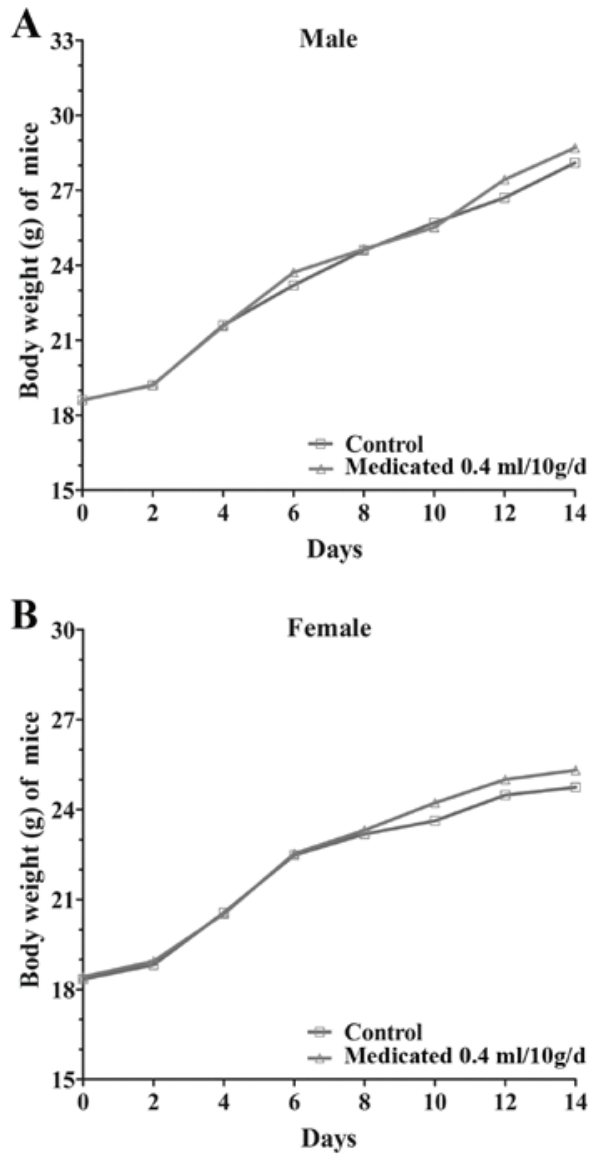

Figure 1. Body weight changes in mice following an acute toxicity assessment and treatment with orally administered jiangu capsules. Body weight changes in (A) male and (B) female mice.

Chronic toxicity study. The chronic toxicity study was conducted over 6.5 months. The $120 \mathrm{SD}$ rats were housed in groups of 10 in plastic cages, segregated by gender. The animals were randomly grouped into one of four groups: A control group and high, medium and low dose groups. Each group consisted of 15 females and 15 males. The weight of each animal was recorded. The jiangu capsule was administered orally for 6 months ( 6 times per week) to the high, medium and low dose groups in the following concentrations: 8, 4 and $2 \mathrm{~g} / \mathrm{kg} /$ day, respectively. During a period of 14 days post treatment, rats were fed with standard commercial diet and tap water and had ad libitum access to feed and drinking water. The control group received distilled water $(20 \mathrm{ml} / \mathrm{kg} / \mathrm{day})$. Following 6 months of treatment, 3 months post-treatment and 14 days of withdrawal without any drug treatment, 10 rats from each group (5 females and 5 males) were sacrificed by cervical dislocation under anesthesia with $20 \%$ urethane (ethyl carbamate; i.p., 1,000 mg/kg; Shanghai Hengyuan Biological Technology Co., Ltd., Shanghai, China). Sacrificed rats were used to assess relevant parameters of hematological and biochemical analysis, urinalysis, systematic anatomy, organ weights and histopathological examination (18).

General condition, body weight changes, and food and water consumption. The general condition of different groups was observed for $1 \mathrm{~h}$ following treatment, then intermittently for $4 \mathrm{~h}$ and thereafter over a period of $24 \mathrm{~h}$. The following assessments were carried out daily using a previously published scale: Pelage was determined by assessing luster; behavior and activity were determined by assessing agility; respiration was determined by assessing if the rats had even breathing; rats were examined for the presence of any abnormal secretions from the eyes, nose and genitals; and the colour of the rats' urine was assessed $(18,19)$. Body weight and food consumption were recorded weekly (20).

Hematological parameters. Blood samples of rats in the four groups were collected following month 3 and 6, and following 14 days of withdrawal and analyzed using a BC-5500 Auto Hematological auto-analyzer (Mindray Medical International, Ltd., Shenzhen, China). The hematological parameters assessed included red blood cell count, mean corpuscular hemoglobin concentration, hematocrit, mean corpuscular hemoglobin, white blood cell (WBC) count, platelet count, haemoglobin and WBC differential count (21).

Biochemical parameters. At the end of 3 and 6 months treatment, and 14 days of withdrawal, urine was collected using a syringe whilst rats were anesthetized with $20 \%$ urethane (Ethyl carbamate; $0.5 \mathrm{ml} / 100 \mathrm{~g}$; Shanghai Hengyuan Biological Technology Co., Ltd). The detection of blood, nitrite, $\mathrm{pH}$, urobilinogen, bilirubin, protein, glucose and ketone bodies in urine was performed using visual eight league test strips (Guangzhou Huadu Gao'erbao Biological Technology Co., Ltd Guangzhou, China). Blood samples were centrifuged at $3,000 \mathrm{x} \mathrm{g}$ for $10 \mathrm{~min}$ at $4^{\circ} \mathrm{C}$. Serum was then analyzed using the CL-8000 automatic biochemistry analyzer (Shimadzu Corporation, Kyoto, Japan) for biochemical parameters. These parameters included blood urea nitrogen, creatinine, cholesterol, total bilirubin, total protein, albumin, glucose, alanine aminotransferase, aminotransferase, alkaline phosphatase, triglyceride and creatine kinase (22).

Systematic anatomy. Immediately following blood collection, all animals were sacrificed by cervical dislocation under $20 \%$ urethane anesthesia $(1,000 \mathrm{mg} / \mathrm{kg}$, Shanghai Hengyuan Biological Technology Co., Ltd) and organs including heart, liver, spleen, lungs, kidneys, adrenal glands, thymus, uterus, ovary, testis, prostate, thyroid, stomach, bladder, pancreas, thoracic cavity and abdominal cavity were removed and observed for gross lesions.

Organ weights. Following organ collection, blood was removed using filter paper and the connective tissue around each organ was cut. Upon sacrifice, the organ weights of all animals were measured using an electronic balance. These organs included heart, liver, spleen, lung, kidney, adrenal gland, thymus, prostate, testis, ovary, brain, uterus and epididymis (23).

Histopathological examination. Careful pathological examinations were performed on the following organs: Heart, liver, spleen, lung, kidney, adrenal gland, thymus, stomach, small intestine, large intestine, thyroid, parathyroid, brain, cerebellum, brainstem, pancreas, uterus, ovary, breast, testis, epididymis, prostate, bone marrow, spinal cord, lymph nodes, esophagus, trachea, pituitary gland, bladder, arteries, sciatic nerve and optic nerve. The aforementioned organ samples were fixed in $10 \%$ formalin for $36 \mathrm{~h}$ at $25^{\circ} \mathrm{C}$, embedded 
A

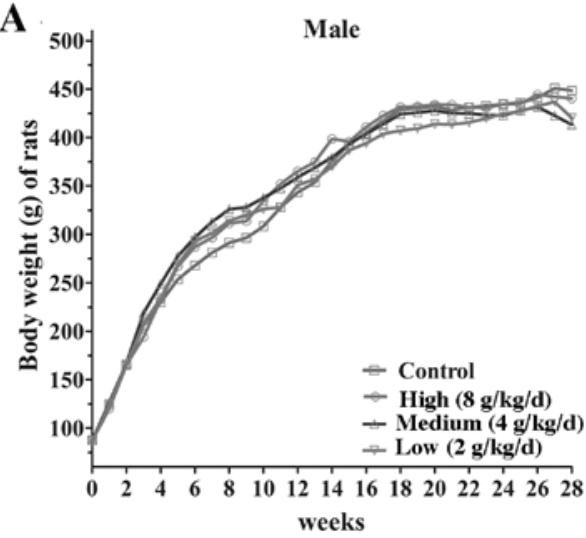

B

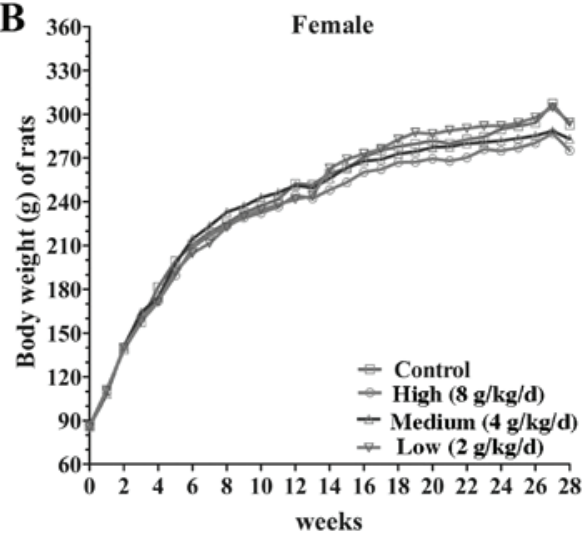

Figure 2. Body weight changes of rats treated with orally administered jiangu capsule over 28 weeks during the chronic toxicity assessment. There were no significant differences among any of the groups. Body weight changes of (A) male and (B) female rats.

in paraffin, sliced into sections $5-\mu \mathrm{m}$ thick, stained with haematoxylin for 5-10 min and then stained with eosin for $1-3$ min at $25^{\circ} \mathrm{C}$. Samples were observed using an optical microscope $(24,25)$.

Statistical analysis. Data were expressed as the mean \pm standard error of the mean and analyzed using one-way analysis of variance followed by least significant difference correction for multiple comparisons tests. All figures were created using GraphPad Prism version 5.01 (GraphPad Software, Inc., La Jolla, CA, USA). P $<0.05$ was considered to indicate a statistically significant difference.

\section{Results}

Acute oral toxicity of jiangu capsule treatment in mice. The highest dose $(16 \mathrm{~g} / \mathrm{kg})$ of orally administered jiangu capsule did not induce any signs of acute toxicity or mortality in mice. Mice in the control and treatment groups had glossy fur, moved with ease and demonstrated normal food intake, urination and defecation during the acute toxicity assessment. The body weight gain of the treated groups was similar to the control group during the study (Fig. 1). Following the experiment, all mice were sacrificed and morphological observations demonstrated that the size and exterior colors of internal organs (including the heart, liver, spleen, lung and kidney) of the treated groups were the same as the control group. The
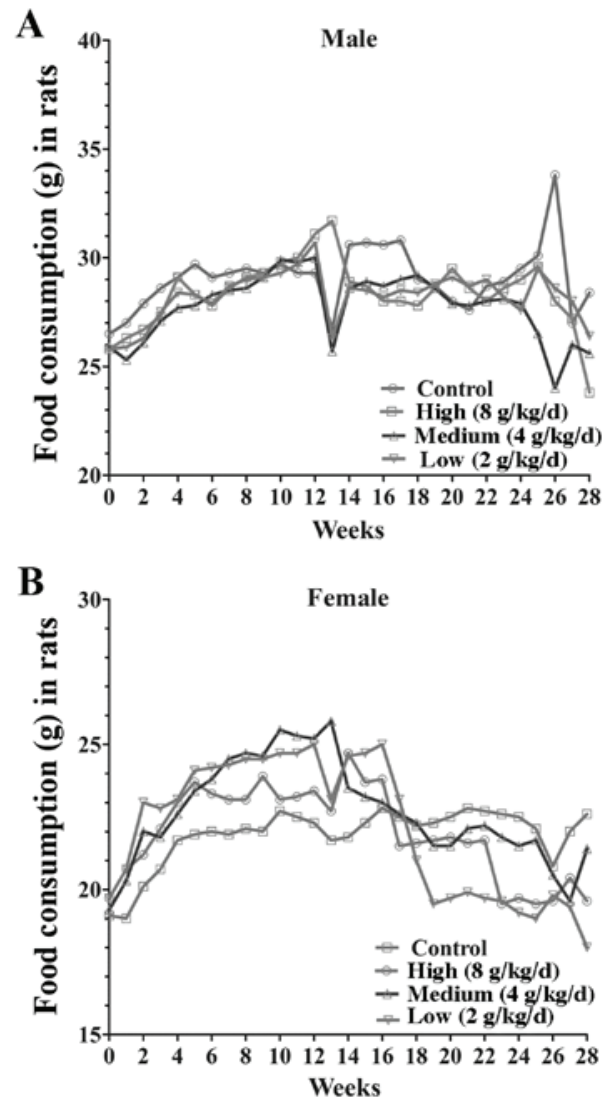

Figure 3. Food consumption changes in rats following treatment with orally administered jiangu capsules over 28 weeks during the chronic toxicity assessment. There were no significant differences among any of the groups. Body weight changes of (A) male and (B) female rats.

results indicated that the maximum dose of jiangu capsule in mice is $\sim 16 \mathrm{~g} / \mathrm{kg}$.

Chronic oral toxicity study of jiangu capsule treatment in $S D$ rats. The general condition, body weight changes, food and water consumption were assessed. No rats succumbed during the 6 months of oral administration. There was no change in appearance, activity or excrement of the treated group when compared with the control group during the chronic toxicity test. There was no significant difference between the body weight gain $(\mathrm{P}>0.05)$ and food consumption of male and female rats at each dose (8, 4, $2 \mathrm{~g} / \mathrm{kg} /$ day; Fig. 2). Following 14 days of withdrawal, there were no significant differences among the groups in body weight gain and food intake (Fig. 3). These results demonstrated that the jiangu capsule exhibits no marked effects on body weight or food consumption in rats.

Hematological parameters. The hematological parameters of groups treated with the jiangu capsule were within the normal physiological ranges (red blood cell count: $7.2-9.6 \times 10^{12} / 1$, hemoglobin: $120-170 \mathrm{~g} / 1$, hematocrit: $35-50 \%$, mean corpuscular hemoglobin: $15-20 \mathrm{pg}$, mean corpuscular hemoglobin concentration: $30-40 \mathrm{~g} / 1$, platelet count: $50-100 \times 10^{9} / 1$, white blood cell count: $6.0-15.0 \times 10^{9} / 1$, lymphocyte: $65-84 \%$, neutrophil: $9-34 \%$, monocyte: $0-5 \%$ ) and the medicated groups exhibited no significant difference when compared with the 
Table II. Effect of chronic oral administration on the hematological parameters of rats following treatment with jiangu capsule.

Treatment period

\begin{tabular}{|c|c|c|c|c|}
\hline Parameter & $\begin{array}{c}\text { Normal } \\
\text { physiological ranges }\end{array}$ & 3 months & 6 months & $\begin{array}{c}14 \text { days of } \\
\text { withdrawal }\end{array}$ \\
\hline \multicolumn{5}{|l|}{ Control } \\
\hline $\mathrm{RBC}, \times 10^{12} / 1$ & $7.2-9.6$ & $8.22 \pm 0.43$ & $8.31 \pm 0.42$ & $8.30 \pm 0.44$ \\
\hline $\mathrm{HGB}, \mathrm{g} / \mathrm{l}$ & $120-170$ & $166.32 \pm 5.26$ & $165.25 \pm 6.10$ & $167.71 \pm 4.75$ \\
\hline НCT, \% & $35-50$ & $45.82 \pm 2.56$ & $46.28 \pm 3.33$ & $45.94 \pm 2.54$ \\
\hline $\mathrm{MCH}, \mathrm{pg}$ & $15-20$ & $20.26 \pm 0.67$ & $19.92 \pm 0.61$ & $20.25 \pm 0.62$ \\
\hline $\mathrm{MCHC}, \mathrm{g} / \mathrm{l}$ & $30-40$ & $363.89 \pm 21.34$ & $358.98 \pm 32.03$ & $366.07 \pm 22.77$ \\
\hline PLT, $x 10^{9} / 1$ & $50-100$ & $723.10 \pm 89.69$ & $713.70 \pm 85.83$ & $702.00 \pm 97.23$ \\
\hline WBC, $\times 10^{9} / 1$ & $6.0-15.0$ & $14.13 \pm 3.70$ & $14.71 \pm 3.52$ & $14.26 \pm 3.33$ \\
\hline Lymphocyte, \% & $65-84$ & $71.50 \pm 5.34$ & $68.70 \pm 4.32$ & $68.50 \pm 3.31$ \\
\hline Neutrophil, \% & $9-34$ & $21.70 \pm 6.55$ & $24.10 \pm 3.31$ & $23.50 \pm 3.44$ \\
\hline Monocyte, \% & $0-5$ & $6.80 \pm 1.87$ & $7.20 \pm 2.66$ & $8.00 \pm 2.40$ \\
\hline \multicolumn{5}{|l|}{ High dose $(8 \mathrm{~g} / \mathrm{kg})$} \\
\hline $\mathrm{RBC}, \mathrm{x} 10^{12} / 1$ & $7.2-9.6$ & $8.25 \pm 0.36$ & $8.18 \pm 0.45$ & $8.30 \pm 0.44$ \\
\hline $\mathrm{HGB}, \mathrm{g} / \mathrm{l}$ & $120-170$ & $166.28 \pm 6.78$ & $167.78 \pm 6.63$ & $167.71 \pm 4.75$ \\
\hline НСТ, \% & $35-50$ & $44.78 \pm 2.71$ & $45.83 \pm 2.88$ & $45.94 \pm 2.54$ \\
\hline $\mathrm{MCH}, \mathrm{pg}$ & $15-20$ & $20.16 \pm 0.25$ & $20.53 \pm 0.53$ & $20.25 \pm 0.62$ \\
\hline MCHC, g/l & $30-40$ & $372.62 \pm 27.79$ & $367.69 \pm 31.70$ & $366.07 \pm 22.77$ \\
\hline PLT, $x 10^{9} / 1$ & $50-100$ & $695.30 \pm 125.38$ & $762.00 \pm 105.99$ & $750.10 \pm 73.88$ \\
\hline WBC, $\times 10^{9} / 1$ & $6.0-15.0$ & $13.64 \pm 4.19$ & $14.70 \pm 3.59$ & $14.40 \pm 3.22$ \\
\hline Lymphocyte, \% & $65-84$ & $70.50 \pm 6.08$ & $67.70 \pm 5.96$ & $67.80 \pm 4.71$ \\
\hline Neutrophil, \% & $9-34$ & $21.60 \pm 7.15$ & $24.40 \pm 2.80$ & $23.40 \pm 2.22$ \\
\hline Monocyte, \% & $0-5$ & $7.90 \pm 2.64$ & $762.00 \pm 105.99$ & $8.80 \pm 4.29$ \\
\hline \multicolumn{5}{|c|}{ Medium dose (4 g/kg) } \\
\hline $\mathrm{RBC}, \times 10^{12} / 1$ & $7.2-9.6$ & $8.07 \pm 0.36$ & $8.21 \pm 0.46$ & $8.33 \pm 0.41$ \\
\hline $\mathrm{HGB}, \mathrm{g} / \mathrm{l}$ & $120-170$ & $162.46 \pm 5.27$ & $165.27 \pm 7.37$ & $164.73 \pm 6.27$ \\
\hline $\mathrm{HCT}, \%$ & $35-50$ & $45.38 \pm 2.06$ & $45.88 \pm 2.98$ & $46.34 \pm 2.99$ \\
\hline $\mathrm{MCH}, \mathrm{pg}$ & $15-20$ & $20.14 \pm 0.56$ & $20.13 \pm 0.33$ & $19.79 \pm 0.56$ \\
\hline MCHC, g/l & $30-40$ & $358.55 \pm 17.20$ & $361.49 \pm 27.19$ & $356.90 \pm 27.85$ \\
\hline PLT, $\times 10^{9} / 1$ & $50-100$ & $711.10 \pm 84.18$ & $776.80 \pm 87.30$ & $716.80 \pm 91.34$ \\
\hline $\mathrm{WBC}, \times 10^{9} / 1$ & $6.0-15.0$ & $14.02 \pm 3.40$ & $13.92 \pm 3.76$ & $14.63 \pm 3.11$ \\
\hline Lymphocyte, \% & $65-84$ & $66.40 \pm 4.97$ & $67.60 \pm 4.40$ & $68.90 \pm 3.63$ \\
\hline Neutrophil, \% & $9-34$ & $25.10 \pm 3.25$ & $24.60 \pm 3.69$ & $21.90 \pm 4.07$ \\
\hline Monocyte, \% & $0-5$ & $8.50 \pm 3.75$ & $776.80 \pm 87.30$ & $9.20 \pm 2.44$ \\
\hline \multicolumn{5}{|l|}{ Low dose (2 g/kg) } \\
\hline $\mathrm{RBC}, \mathrm{x} 10^{12} / 1$ & $7.2-9.6$ & $8.14 \pm 0.29$ & $8.25 \pm 0.38$ & $8.37 \pm 0.39$ \\
\hline $\mathrm{HGB}, \mathrm{g} / \mathrm{l}$ & $120-170$ & $165.47 \pm 6.17$ & $166.45 \pm 5.95$ & $166.95 \pm 4.59$ \\
\hline $\mathrm{HCT}, \%$ & $35-50$ & $45.94 \pm 2.47$ & $45.99 \pm 2.71$ & $46.08 \pm 2.79$ \\
\hline $\mathrm{MCH}, \mathrm{pg}$ & $15-20$ & $20.36 \pm 0.94$ & $20.18 \pm 0.36$ & $19.96 \pm 0.46$ \\
\hline MCHC, g/l & $30-40$ & $361.26 \pm 26.19$ & $363.33 \pm 29.13$ & $363.68 \pm 26.71$ \\
\hline PLT, $x 10^{9} / 1$ & $50-100$ & $764.20 \pm 101.53$ & $711.10 \pm 86.98$ & $716.00 \pm 87.36$ \\
\hline WBC, $\times 10^{9} / 1$ & $6.0-15.0$ & $14.28 \pm 3.16$ & $14.75 \pm 3.76$ & $13.95 \pm 3.47$ \\
\hline Lymphocyte, \% & $65-84$ & $70.60 \pm 2.72$ & $71.10 \pm 5.86$ & $68.20 \pm 4.16$ \\
\hline Neutrophil, \% & $9-34$ & $23.80 \pm 3.94$ & $21.20 \pm 4.39$ & $24.60 \pm 2.84$ \\
\hline Monocyte, \% & $0-5$ & $5.60 \pm 2.22$ & $711.10 \pm 86.98$ & $7.20 \pm 3.16$ \\
\hline
\end{tabular}

Values are expressed as mean \pm standard error of the mean, $\mathrm{n}=10$. P>0 .05. RBC, red blood cell; HGB, hemoglobin; HCT, hematocrit; MCH, mean corpuscular hemoglobin; MCHC, mean corpuscular hemoglobin concentration; PLT, platelet count; WBC, white blood cell. 
Table III. Effect of chronic oral administration of jiangu capsule on the biochemical parameters of rats.

\begin{tabular}{lrrr}
\hline & \multicolumn{3}{c}{ Treatment period } \\
\cline { 2 - 4 } $\begin{array}{l}\text { Biochemical } \\
\text { parameter }\end{array}$ & 3 months & 6 months & $\begin{array}{c}14 \text { days } \\
\text { withdrawal }\end{array}$ \\
\hline Control & & \\
Total proteins, & $70.43 \pm 3.09$ & $70.07 \pm 3.32$ & $69.78 \pm 4.58$ \\
g/l & & & \\
Albumin, g/l & $33.82 \pm 3.76$ & $33.21 \pm 3.32$ & $32.08 \pm 3.56$ \\
ALT, U/1 & $41.01 \pm 4.86$ & $42.87 \pm 4.31$ & $41.50 \pm 5.12$ \\
AST, U/1 & $56.62 \pm 4.29$ & $63.03 \pm 5.03$ & $57.88 \pm 3.37$ \\
ALP, U/1 & $84.49 \pm 9.34$ & $83.93 \pm 8.79$ & $76.85 \pm 13.92$ \\
Glucose, & $7.01 \pm 0.79$ & $6.72 \pm 0.86$ & $7.22 \pm 0.64$ \\
mmol/l & & & \\
Total bilirubin, & $4.86 \pm 1.29$ & $5.03 \pm 1.44$ & $6.30 \pm 1.30$ \\
$\mu$ mol/l & & & \\
Urea, mmol/l & $7.65 \pm 0.76$ & $8.41 \pm 0.73$ & $8.12 \pm 0.91$ \\
Creatinine, & $69.43 \pm 8.94$ & $72.90 \pm 9.71$ & $71.87 \pm 9.99$ \\
$\mu$ mol/l & & & \\
TC, mmol/1 & $1.77 \pm 0.30$ & $1.59 \pm 0.25$ & $1.62 \pm 0.22$ \\
TG, mmol/1 & $1.04 \pm 0.17$ & $0.95 \pm 0.21$ & $0.96 \pm 0.16$ \\
CK, U/1 & $579.00 \pm 81.23$ & $679.90 \pm 74.04$ & $663.60 \pm 66.30$ \\
& & &
\end{tabular}

High dose

$(8 \mathrm{~g} / \mathrm{kg})$

$\begin{array}{lrrc}\text { Total proteins, } & 69.48 \pm 3.79 & 69.89 \pm 4.05 & 70.06 \pm 3.62 \\ \text { g/l } & & & \\ \text { Albumin, g/l } & 31.91 \pm 4.06 & 31.05 \pm 4.50 & 70.06 \pm 3.62 \\ \text { ALT, U/l } & 44.96 \pm 4.30 & 43.62 \pm 5.26 & 70.06 \pm 3.62 \\ \text { AST, U/1 } & 56.39 \pm 5.48 & 57.98 \pm 5.50 & 70.06 \pm 3.62 \\ \text { ALP, U/1 } & 84.43 \pm 9.88 & 4.10 \pm 1.12 & 84.14 \pm 11.59 \\ \text { Glucose, } & 7.27 \pm 0.80 & 4.10 \pm 1.12 & 7.10 \pm 0.98 \\ \text { mmol/l } & & & \\ \text { Total bilirubin, } & 4.97 \pm 1.44 & 4.10 \pm 1.12 & 7.06 \pm 1.57 \\ \mu \text { mol/l } & & & \\ \text { Urea, mmol/l } & 8.02 \pm 0.78 & 8.46 \pm 0.74 & 8.48 \pm 0.96 \\ \text { Creatinine, } & 74.10 \pm 9.32 & 71.25 \pm 8.62 & 70.70 \pm 9.98 \\ \mu \text { mol/l } & & & \\ \text { TC, mmol/1 } & 1.63 \pm 0.30 & 1.63 \pm 0.26 & 1.64 \pm 0.28 \\ \text { TG, mmol/1 } & 1.05 \pm 0.23 & 0.98 \pm 0.22 & 0.99 \pm 0.21 \\ \text { CK, U/l } & 650.70 \pm 75.81 & 695.10 \pm 77.53 & 655.10 \pm 64.84\end{array}$

Medium dose

$(4 \mathrm{~g} / \mathrm{kg})$

Total proteins, $\mathrm{g} / \mathrm{l}$

Albumin, g/1

$69.18 \pm 2.29 \quad 68.37 \pm 3.40$

$69.76 \pm 3.56$

ALT, U/1

$31.59 \pm 3.14$

AST, U/1

ALP, U/1

Glucose,

$\mathrm{mmol} / \mathrm{l}$

Total bilirubin, $\quad 4.49 \pm 1.19$

$30.83 \pm 3.31$

$32.17 \pm 3.99$

$42.85 \pm 4.96 \quad 43.28 \pm 4.98$

$57.36 \pm 2.94 \quad 58.62 \pm 4.90$

$75.25 \pm 12.78 \quad 81.69 \pm 8.37$

$45.84 \pm 6.52$

$59.02 \pm 4.43$

$83.04 \pm 11.02$

$6.92 \pm 0.89$

$6.96 \pm 1.01$

$6.77 \pm 1.41$

$6.46 \pm 1.74$

$\mu \mathrm{mol} / 1$

Urea, $\mathrm{mmol} / \mathrm{l}$
Table III. Continued.

Treatment period

\begin{tabular}{|c|c|c|c|}
\hline $\begin{array}{l}\text { Biochemical } \\
\text { parameter }\end{array}$ & 3 months & 6 months & $\begin{array}{c}14 \text { days } \\
\text { withdrawal }\end{array}$ \\
\hline $\begin{array}{l}\text { Creatinine, } \\
\mu \mathrm{mol} / 1\end{array}$ & $69.39 \pm 5.43$ & $74.23 \pm 9.82$ & $73.39 \pm 9.34$ \\
\hline $\mathrm{TC}, \mathrm{mmol} / \mathrm{l}$ & $1.60 \pm 0.27$ & $1.64 \pm 0.24$ & $1.65 \pm 0.24$ \\
\hline $\mathrm{TG}, \mathrm{mmol} / \mathrm{l}$ & $1.00 \pm 0.19$ & $1.03 \pm 0.15$ & $1.04 \pm 0.15$ \\
\hline $\mathrm{CK}, \mathrm{U} / \mathrm{l}$ & $654.70 \pm 77.43$ & $703.90 \pm 75.05$ & $662.50 \pm 58.18$ \\
\hline \multicolumn{4}{|l|}{$\begin{array}{l}\text { Low dose } \\
(2 \mathrm{~g} / \mathrm{kg})\end{array}$} \\
\hline $\begin{array}{l}\text { Total proteins, } \\
\mathrm{g} / 1\end{array}$ & $69.56 \pm 3.75$ & $69.80 \pm 2.67$ & $69.19 \pm 3.20$ \\
\hline Albumin, g/l & $31.29 \pm 3.49$ & $31.94 \pm 3.85$ & $31.68 \pm 4.00$ \\
\hline ALT, U/l & $42.76 \pm 4.55$ & $42.10 \pm 5.54$ & $42.94 \pm 5.44$ \\
\hline AST, U/l & $55.68 \pm 4.60$ & $57.56 \pm 4.87$ & $57.21 \pm 2.41$ \\
\hline GGT, U/1 & $4.50 \pm 1.23$ & $3.96 \pm 1.27$ & $4.89 \pm 1.32$ \\
\hline ALP, U/1 & $83.19 \pm 8.67$ & $81.79 \pm 10.59$ & $77.75 \pm 8.15$ \\
\hline $\begin{array}{l}\text { Glucose, } \\
\mathrm{mmol} / \mathrm{l}\end{array}$ & $7.18 \pm 0.71$ & $7.32 \pm 0.77$ & $6.93 \pm 0.81$ \\
\hline $\begin{array}{l}\text { Total bilirubin, } \\
\mu \mathrm{mol} / 1\end{array}$ & $4.09 \pm 1.45$ & $5.91 \pm 1.25$ & $5.40 \pm 1.16$ \\
\hline Urea, mmol/l & $8.29 \pm 0.46$ & $8.47 \pm 0.76$ & $8.46 \pm 0.77$ \\
\hline $\begin{array}{l}\text { Creatinine, } \\
\mu \mathrm{mol} / 1\end{array}$ & $75.66 \pm 5.53$ & $71.50 \pm 8.40$ & $73.25 \pm 8.71$ \\
\hline $\mathrm{TC}, \mathrm{mmol} / \mathrm{l}$ & $1.66 \pm 0.27$ & $1.52 \pm 0.25$ & $1.65 \pm 0.22$ \\
\hline $\mathrm{TG}, \mathrm{mmol} / \mathrm{l}$ & $0.98 \pm 0.18$ & $0.96 \pm 0.19$ & $1.06 \pm 0.16$ \\
\hline $\mathrm{CK}, \mathrm{U} / \mathrm{l}$ & $663.70 \pm 73.11$ & $673.10 \pm 69.67$ & $684.40 \pm 65.24$ \\
\hline
\end{tabular}

Values are expressed as mean \pm standard error of the mean, $n=10$. $\mathrm{P}>0.05$. ALT, alanine aminotransferase; AST, aminotransferase; ALP, alkaline phosphate; TC, total cholesterol; TG, triglyceride; CK, creatine kinase.

control group (Table II; P $>0.05$ ). The data suggested that jiangu capsule treatment exerted no discernable effect on hematological parameters.

Biochemical parameters. The biochemical values of rats in the jiangu capsule-treated and control groups are presented in Table III. The parameter index of the treated group was within the normal physiological range. There were no significant differences in biochemical parameters between the control and medicated groups ( $\mathrm{P}>0.05$; Table III). Urine from all the animals was a transparent liquid with a light yellow color, and no abnormal changes in the urinalysis results were observed in the treatment group compared with the control group. The results suggest that jiangu capsule treatment has no significant impact on biochemical parameters or urine composition.

Systematic anatomy. Internal organs were observed by the naked eye. No effusion was observed in the pleural or 
Table IV. Effect of chronic oral administration of jiangu capsule on organ weights of rats.

\begin{tabular}{|c|c|c|c|}
\hline \multirow[b]{2}{*}{ Parameter } & \multicolumn{3}{|c|}{ Treatment period } \\
\hline & 3 months & 6 months & $\begin{array}{l}14 \text { days of } \\
\text { withdrawal }\end{array}$ \\
\hline \multicolumn{4}{|l|}{ Control } \\
\hline Heart & $0.33 \pm 0.03$ & $0.36 \pm 0.05$ & $0.36 \pm 0.06$ \\
\hline Liver & $2.93 \pm 0.24$ & $2.64 \pm 0.29$ & $2.39 \pm 0.24$ \\
\hline Spleen & $0.21 \pm 0.03$ & $0.21 \pm 0.05$ & $0.16 \pm 0.03$ \\
\hline Lung & $0.78 \pm 0.21$ & $0.52 \pm 0.14$ & $0.65 \pm 0.10$ \\
\hline Kidney & $0.68 \pm 0.05$ & $0.63 \pm 0.08$ & $0.61 \pm 0.06$ \\
\hline Brain & $0.65 \pm 0.11$ & $0.59 \pm 0.14$ & $0.56 \pm 0.12$ \\
\hline Uterus $^{\mathrm{a}}$ & $0.21 \pm 0.04$ & $0.27 \pm 0.06$ & $0.23 \pm 0.04$ \\
\hline Testicles $^{\mathrm{a}}$ & $0.82 \pm 0.08$ & $0.73 \pm 0.03$ & $0.74 \pm 0.06$ \\
\hline Epididymis $^{\mathrm{a}}$ & $0.45 \pm 0.08$ & $0.37 \pm 0.03$ & $0.42 \pm 0.05$ \\
\hline Prostate $^{a}$ & $0.09 \pm 0.01$ & $0.16 \pm 0.02$ & $0.16 \pm 0.05$ \\
\hline Adrenal glands & $22.45 \pm 6.98$ & $24.12 \pm 10.04$ & $20.91 \pm 7.88$ \\
\hline Thymus & $115.83 \pm 25.27$ & $86.41 \pm 20.02$ & $93.67 \pm 20.78$ \\
\hline Ovaries $^{\mathrm{a}}$ & $49.91 \pm 8.47$ & $45.20 \pm 8.12$ & $52.81 \pm 11.42$ \\
\hline \multicolumn{4}{|l|}{$\begin{array}{l}\text { High dose } \\
(8 \mathrm{~g} / \mathrm{kg})\end{array}$} \\
\hline Heart & $0.34 \pm 0.04$ & $0.36 \pm 0.04$ & $0.36 \pm 0.05$ \\
\hline Liver & $2.76 \pm 0.33$ & $2.88 \pm 0.41$ & $2.43 \pm 0.24$ \\
\hline Spleen & $0.20 \pm 0.04$ & $0.22 \pm 0.06$ & $0.17 \pm 0.06$ \\
\hline Lung & $0.65 \pm 0.21$ & $0.53 \pm 0.06$ & $0.64 \pm 0.12$ \\
\hline Kidney & $0.69 \pm 0.06$ & $0.64 \pm 0.05$ & $0.61 \pm 0.06$ \\
\hline Brain & $0.67 \pm 0.15$ & $0.57 \pm 0.13$ & $0.56 \pm 0.12$ \\
\hline Uterus $^{\mathrm{a}}$ & $0.27 \pm 0.10$ & $0.25 \pm 0.02$ & $0.23 \pm 0.03$ \\
\hline Testicles $^{\mathrm{a}}$ & $0.77 \pm 0.04$ & $0.72 \pm 0.03$ & $0.73 \pm 0.13$ \\
\hline Epididymis $^{\mathrm{a}}$ & $0.40 \pm 0.08$ & $0.36 \pm 0.03$ & $0.43 \pm 0.05$ \\
\hline Prostate $^{a}$ & $0.10 \pm 0.01$ & $0.17 \pm 0.02$ & $0.17 \pm 0.03$ \\
\hline Adrenal glands & $19.52 \pm 7.63$ & $28.18 \pm 9.19$ & $26.44 \pm 10.69$ \\
\hline Thymus & $104.95 \pm 34.19$ & $77.25 \pm 25.16$ & $79.86 \pm 24.57$ \\
\hline Ovaries $^{\mathrm{a}}$ & $41.63 \pm 1.46$ & $46.78 \pm 24.26$ & $48.20 \pm 11.34$ \\
\hline \multicolumn{4}{|l|}{$\begin{array}{l}\text { Medium dose } \\
(4 \mathrm{~g} / \mathrm{kg})\end{array}$} \\
\hline Heart & $0.34 \pm 0.04$ & $0.36 \pm 0.05$ & $0.36 \pm 0.04$ \\
\hline Liver & $2.85 \pm 0.26$ & $2.82 \pm 0.66$ & $2.52 \pm 0.31$ \\
\hline Spleen & $0.24 \pm 0.06$ & $0.21 \pm 0.06$ & $0.18 \pm 0.04$ \\
\hline Lung & $0.82 \pm 0.25$ & $0.53 \pm 0.07$ & $0.62 \pm 0.07$ \\
\hline Kidney & $0.69 \pm 0.08$ & $0.62 \pm 0.05$ & $0.61 \pm 0.09$ \\
\hline Brain & $0.68 \pm 0.15$ & $0.58 \pm 0.13$ & $0.58 \pm 0.12$ \\
\hline Uterus $^{\mathrm{a}}$ & $0.25 \pm 0.07$ & $0.23 \pm 0.03$ & $0.23 \pm 0.05$ \\
\hline Testicles $^{\mathrm{a}}$ & $0.77 \pm 0.07$ & $0.74 \pm 0.10$ & $0.74 \pm 0.17$ \\
\hline Epididymis $^{\mathrm{a}}$ & $0.39 \pm 0.07$ & $0.39 \pm 0.04$ & $0.43 \pm 0.09$ \\
\hline Prostate $^{\mathrm{a}}$ & $0.08 \pm 0.02$ & $0.17 \pm 0.04$ & $0.16 \pm 0.02$ \\
\hline Adrenal glands & $23.41 \pm 9.52$ & $21.53 \pm 13.22$ & $24.37 \pm 9.77$ \\
\hline Thymus & $103.43 \pm 19.46$ & $74.74 \pm 20.55$ & $87.54 \pm 24.60$ \\
\hline Ovaries $^{\mathrm{a}}$ & $48.14 \pm 9.50$ & $47.83 \pm 11.14$ & $52.70 \pm 6.07$ \\
\hline
\end{tabular}

Low dose

$(2 \mathrm{~g} / \mathrm{kg})$

$\begin{array}{llll}\text { Heart } & 0.34 \pm 0.06 & 0.37 \pm 0.04 & 0.36 \pm 0.06 \\ \text { Liver } & 2.76 \pm 0.28 & 2.67 \pm 0.47 & 2.53 \pm 0.22\end{array}$

Table IV. Continued.

\begin{tabular}{|c|c|c|c|}
\hline \multirow[b]{2}{*}{ Parameter } & \multicolumn{3}{|c|}{ Treatment period } \\
\hline & 3 months & 6 months & $\begin{array}{l}14 \text { days of } \\
\text { withdrawal }\end{array}$ \\
\hline \multicolumn{4}{|l|}{$\begin{array}{l}\text { Low dose } \\
(2 \mathrm{~g} / \mathrm{kg})\end{array}$} \\
\hline Spleen & $0.23 \pm 0.05$ & $0.21 \pm 0.04$ & $0.17 \pm 0.03$ \\
\hline Lung & $0.71 \pm 0.18$ & $0.52 \pm 0.07$ & $0.66 \pm 0.05$ \\
\hline Kidney & $0.70 \pm 0.06$ & $0.63 \pm 0.04$ & $0.62 \pm 0.05$ \\
\hline Brain & $0.68 \pm 0.18$ & $0.57 \pm 0.11$ & $0.57 \pm 0.11$ \\
\hline Uterus $^{\mathrm{a}}$ & $0.23 \pm 0.09$ & $0.24 \pm 0.04$ & $0.24 \pm 0.06$ \\
\hline Testicles $^{\mathrm{a}}$ & $0.84 \pm 0.10$ & $0.73 \pm 0.10$ & $0.75 \pm 0.05$ \\
\hline Epididymis $^{\mathrm{a}}$ & $0.42 \pm 0.11$ & $0.39 \pm 0.03$ & $0.44 \pm 0.04$ \\
\hline Prostate $^{a}$ & $0.10 \pm 0.03$ & $0.17 \pm 0.02$ & $0.16 \pm 0.03$ \\
\hline Adrenal glands & $25.32 \pm 9.61$ & $27.24 \pm 6.18$ & $21.96 \pm 6.31$ \\
\hline Thymus & $111.38 \pm 45.44$ & $81.62 \pm 25.89$ & $92.41 \pm 18.23$ \\
\hline Ovaries $^{\mathrm{a}}$ & $46.04 \pm 8.46$ & $51.38 \pm 7.02$ & $49.98 \pm 6.68$ \\
\hline
\end{tabular}

Values are expressed as mean \pm standard error of the mean, ${ }^{\mathrm{a}} \mathrm{n}=5$, the remaining are all $n=10$. $P>0.05$.

peritoneal cavities and no abnormal colors or morphological changes were identified in the organs of any rat.

Organ weights. The organ weights of the rats medicated with the jiangu capsule and the control group is presented in Table IV. There were no significant differences when compared with the control group during the study $(\mathrm{P}>0.05)$. The results demonstrate that jiangu capsule treatment does not influence organ weight.

Histopathological examination. No histopathological changes were observed in the organs of all rats that were treated with the jiangu capsule. The aforementioned results indicate that the jiangu capsule did not exert an effect on the body weight, food intake, hematological parameters, urine composition, biochemical parameters, systematic anatomy, histopathological parameters or the weight of internal organs of rats that underwent the chronic study.

\section{Discussion}

Traditional herbal medicine serves an important role in complementary and alternative medicine. Saussureae involucratae Flos has been widely applied as a clinical treatment for thousands of years (26). The jiangu capsule is composed of 12 types of natural medicine and has been used to treat a number of conditions, including arthralgia, cold and sore muscles, stiff limbs, inconvenient flexion and activity limitation caused by osteoarthritis $(27,28)$. However, there is a little information regarding jiangu capsule treatment in relation to toxicity. Therefore, the present study aimed to assess the toxicology and identify the safe dosage of the jiangu capsule prior to clinical trials. 
Hematological and serum biochemical parameters are important markers of the physiological and pathological state of the blood. Thus, variations in these parameters may indicate toxicity associated with the compound being assessed. In the chronic study, oral administration for 6 months did not lead to any significant differences in the hematological and biochemical parameters of the treatment groups when compared with controls. These results suggest that the jiangu capsule has no significant toxicological effects on the hemopoietic system.

Urinalysis is known to be the first sign of kidney or urinary tract diseases and may offer important clues regarding the nature of pathological processes (29). The current study did not detect any adverse changes in urine routines when comparing the treatment and control groups. This indicates that the jiangu capsule does not induce toxic effects on the kidney or urinary systems. Assessment of the internal organ weights and histopathological examination of the treatment groups identified no abnormalities and the results were similar to those obtained from the control group. Differences in the organ weights of the two groups were not significant and were not considered to be associated with jiangu treatment. The results obtained therefore indicate that the jiangu capsule is relatively non-toxic.

In the acute toxicity test, the highest oral dose of the jiangu capsule (concentration, 40\%) administered was $16 \mathrm{~g} / \mathrm{kg} / \mathrm{day}$ (yield of extract of the raw material, $47.04 \mathrm{~g} / \mathrm{kg}$ ), which is equal to a 356 -fold increase of the recommended clinical dosage for a $60 \mathrm{~kg}$ human. The mice treated with the jiangu capsule exhibited unlimited movement, normal food consumption and normal excretion throughout the experiment. The acute study demonstrated that there were no adverse changes or mortality in mice following jiangu administration.

In the chronic toxicity study, there were no visible signs of morbidity or mortality in rats that received the highest dose of $8 \mathrm{~g} / \mathrm{kg} / \mathrm{day}$ (yield of extract of the raw material $47.04 \mathrm{~g} / \mathrm{kg}$ ). The highest dose is equivalent to $178 \mathrm{x}$ of the proposed clinical dosage for a $60 \mathrm{~kg}$ human. There were no changes in the general condition, hematological and biochemical values, urinalysis, systematic anatomy, organ weights or histopathological examination in the treatment groups compared with the control group. The results of the chronic study demonstrated that the jiangu capsule does not produce long-term or delayed toxicity in rats following administration.

In conclusion, the present study demonstrated that high doses of jiangu capsule are relatively safe in rats and mice, due to no fatalities occurring or abnormal results in acute and chronic toxicity detected following assessments. Therefore, the jiangu capsule may be used for oral administration without toxic effects. The jiangu capsule may possess the potential to treat diseases including arthralgia, cold and sore muscles, stiff limbs, inconvenient flexion and activity limitation caused by different types of osteoarthritis.

\section{Acknowledgements}

The present study was supported by funds from the National Natural Science Foundation of China (grant no. 81170176), the Scientific Research Foundation for the Returned Overseas Chinese Scholars, the State Education Ministry (grant no. 2012-08), Shaanxi Province Science and Technology Plan Project (grant no. 2014KTCL03-10), and the Ministry of Science and Technology and Technological Special Project for 'Significant New Drugs Development' (grant no. 2011ZX09401-308-32).

\section{References}

1. Song LR: China Herbal. 1st edition. Shanghai Science and Technology Publisher, Shanghai, 1999 (In Chinese).

2. Jung JH, Kim Y, Lee CO, Kang SS, Park JH and Im KS: Cytotoxic constituents of Saussurea lappa. Arch Pharm Res 21: 153-156, 1998.

3. Zheng RL, Liu GS, Xing GX, Jia ZJ, Du M and Tan LQ: Free radical scavenging and antifatigue activities of Saussurea involucrate polysaccharides. Zhongguo Yao Li Xue Bao 14 (Suppl): S47-S49, 1993 (In Chinese).

4. Su KY, Yu CY, Chen YP, Hua KF and Chen YL: 3,4-Dihydroxytoluene, a metabolite of rutin, inhibits inflammatory responses in lipopolysaccharide-activated macrophages by reducing the activation of $\mathrm{NF}-\kappa \mathrm{B}$ signaling. BMC Complement Altern Med 14: 21, 2014.

5. Ma HP, Fan PC, Jing LL, Yao J, He XR, Yang Y, Chen KM and Jia ZP: Anti-hypoxic activity at simulated high altitude was isolated in petroleum ether extract of Saussurea involucrate. J Ethnopharmacol 137: 1510-1515, 2011.

6. Zhao Li and Wang XL: Research on chemical composition of pharmacology and its clinic application of Saussurea Involucrata. J Southwest Univ Nationalities 29: 424-428, 2003 (In Chinese).

7. Singh RP, Chidambara Murthy KN and Jayapakasha GK: Studies on the antioxidant activity of pomegranate (Punica granatum) peel and seed extracts using in vitro models. J Agric Food Chem 50: 81-86, 2002.

8. Wu HF, Ding LS, Wang H and Zhang XF: Advances in the research of phytochemistry and pharmacology of meconopsis vig. Nat Prod Res Dev 23: 163-168, 2011 (In Chinese).

9. Yu F, Yu F, Li R and Wang R: Inhibitory effects of the Gentiana macrophylla (Gentianaceae) extract on rheumatoid arthritis of rats. J Ethnopharmacol 95: 77-81, 2004.

10. Jain S, Sherlekar B and Barik R: Evaluation of antioxidant potential of Tianspora Cordifolia and Tinospaora Sinensis. Int J Clin Pharmacol Res 1: 122-128, 2010.

11. Rodrigues TG, Fernandes A Jr, Sousa JP, Bastos JK and Sforcin JM: In vitro and in vivo effects of clove on proinflammatory cytokines production by macrophages. Nat Prod Res 23: 319-326, 2009.

12. Liu B, Gong HX, Xiao XF and Ye GF: Advances in studies on chemical constituents of Cassia Semen and their pharmacological activities. Drug Eval Res 33: 312-315, 2010 (In Chinese).

13. Lee SH, Lee SY, Son DJ, Lee H, Yoo HS, Song S, Oh KW, Han DC, Kwon BM and Hong JT: Inhibitory effect of 2'-hydroxyeinnamadehyde on nitric oxide production through inhibition of NF-kappa B activation in RAW 264.7 cells. Biochem Pharmacol 69: 791-799, 2005.

14. Xu RY and Tong SH: The research progress of the chemical components and pharmacological effects of safflower. Chin Pharmaceuticals 19: 86-87, 2010 (In Chinese).

15. Kim GY, Lee JY, Lee JO, Ryu CH, Choi BT, Jeong YK, Lee KW, Jeong SC and Choi YH: Partial characterization and immunostimulatory effect of a novel polysaccharide-protein complex extracted from Phellinus linteus. Biosci Biotechnol Biochem 70: 1218-1226, 2006.

16. Yin H: Studies on chemical constituents, pharmacological activities and clinical application of Saussureae Involucratae Herba. J Qiqihar Univ Med 34: 1010-1012, 2013 (In Chinese).

17. He ZM, Li GP, Zhu DS and Lu SM: Guidelines of Management and Use of Laboratory Animals. Science press, Beijing, 2016 (In Chinese).

18. Qi C: The Methodology of Traditional Chinese Medicine Pharmacology Research. 2nd edition. People's Medical Publishing House, Beijing, 2006 (In Chinese).

19. Wei W, Wu XM and Li YJ: Experimental Methodology of Pharmacology. 4th edition. People's Medical Publishing House, Beijing, 2010 (In Chinese).

20. Mu LH, Huang ZX, Liu P, Hu Y and Gao Y: Acute and subchronic oral toxicity assessment of the herbal formula Kai-Xin-San. J Ethnopharmacol 138: 351-357, 2011.

21. Chiranthanut N, Teekachunhatean S, Panthong A, Khonsung P, Kanjanapothi D and Lertprasertsuk N: Toxicity evaluation of standardized extract of Gynostemma pentaphyllum Makino. J Ethnopharmacol 149: 228-234, 2013. 
22. Ha H, Lee JK, Lee HY, Seo CS, Kim JH, Lee MY, Koh WS and Shin HK: Evaluation of safety of the herbal formula Ojeok-san: Acute and sub-chronic toxicity studies in rats. J Ethnopharmacol 131: 410-416, 2010.

23. Ezeja MI, Anaga AO and Asuzu IU: Acute and sub-chronic toxicity profile of methanol leaf extract of Gouania longipetala in rats. J Ethnopharmacol 151: 1155-1164, 2014.

24. Bancroft John D and Gamble Marilyn: Theory and practice of histological techniques, sixth edition Churchill Livingstone, USA, 2007

25. Tahraoui A, Israili ZH and Lyoussi B: Acute and sub-chronic toxicity of a lyophilized aqueous extract of Centaurium erythraea in rodents. J Ethnopharmacol 132: 48-55, 2010.
26. Chik WI, Zhu L, Fan LL, Yi T, Zhu GY, Gou XJ, Tang YN, Xu J, Yeung WP, Zhao ZZ, et al: Saussurea involucrata: A review of the botany, phytochemistry and ethnopharmacology of a rare traditional herbal medicine. J Ethnopharmacol 172: 44-60, 2015.

27. Chinese Pharmacopoeia Commission: Pharmacopeia of the People's Republic of China. vol 1. Chemical Industry Press, Beijing, pp50-51, 2010.

28. National Institutes for Food and Drug Control: Zhongguo Minzu Yaozhi. People's Medical Publishing House, Beijing, pp448-449, 1984.

29. Shao C, Wang Y and Gao Y: Applications of urinary proteomics in biomarker discovery. Sci China Life Sci 54: 409-417, 2011. 